\title{
THE STRONG LEFSCHETZ PROPERTY IN CODIMENSION TWO
}

\author{
DAVID COOK II
}

\begin{abstract}
Every artinian quotient of $K[x, y]$ has the strong Lefschetz property if $K$ is a field of characteristic zero or is an infinite field whose characteristic is greater than the regularity of the quotient. We improve this bound in the case of monomial ideals. Using this we classify when both bounds are sharp. Moreover, we prove that the artinian quotient of a monomial ideal in $K[x, y]$ always has the strong Lefschetz property, regardless of the characteristic of the field, exactly when the ideal is lexsegment. As a consequence, we describe a family of non-monomial complete intersections that always have the strong Lefschetz property.
\end{abstract}

1. Introduction. Let $K$ be an infinite field of arbitrary characteristic, and let $I$ be a homogeneous artinian ideal in $S=K\left[x_{1}, \ldots, x_{n}\right]$. The quotient $S / I$ has the strong Lefschetz property if there exists a linear form $\ell \in[S / I]_{1}$ such that, for all integers $d \geq 0$ and $t \geq 1$, the map $\times \ell^{t}:[S / I]_{d} \rightarrow[S / I]_{d+t}$ has maximal rank. In this case, $\ell$ is a strong Lefschetz element of $S / I$. If the maps have maximal rank for $t=1$ for all $d \geq 0$, then $S / I$ has the weak Lefschetz property, and $\ell$ is a weak Lefschetz element of $S / I$.

The Lefschetz properties have been studied extensively; see the recent survey by Migliore and Nagel [17] and the references contained therein. The interest in these properties largely stems from constraints on the Hilbert functions of quotients that have the weak or strong Lefschetz property (see, e.g., $[\mathbf{2}, \mathbf{9}, \mathbf{1 8}]$ ).

Until recently, most results have focused on characteristic zero or on at least three variables. For artinian quotients of $K[x, y]$, this is not without reason: the weak Lefschetz property always holds, regardless of characteristic. This was explicitly proven for characteristic zero by

2010 AMS Mathematics subject classification. Primary 13A35, 13E10.

Keywords and phrases. Strong Lefschetz property, positive characteristic, lexsegment ideals.

Received by the editors on June 11, 2013, and in revised form on July 17, 2013. DOI:10.1216/JCA-2014-6-3-323 
Harima, et al. in [9, Proposition 4.4] (see the note following the next theorem for more on the characteristic zero case). It was proven for arbitrary characteristic by Migliore and Zanello in [18, Corollary 7], though it was not specifically stated therein as noted by Li and Zanello in [12, Remark 2.6] (see also [7]).

Theorem 1.1. [18, Corollary 7]. Every artinian ideal in $K[x, y]$ has the weak Lefschetz property, regardless of the characteristic of $K$.

Further still, the strong Lefschetz property is known to hold when the characteristic is zero or greater than the regularity of the quotient.

Theorem 1.2. Let $I$ be a homogeneous artinian ideal in $R=K[x, y]$, where $K$ is a field of characteristic $p \geq 0$. The quotient $R / I$ has the strong Lefschetz property if $p=0$ or $p>\operatorname{reg} R / I$.

This result has a varied history. The characteristic zero part was first explicitly given by Harima, et al. [9, Proposition 4.4]. Their proof relies on the generic initial ideal being strongly-stable. Recall that the generic initial ideal is strongly-stable in characteristic zero but also in characteristics larger than the largest exponent of a minimal generator of the ideal (see, e.g., [10, Proposition 4.2.4(b)]). Hence, the proof of [9, Proposition 4.4] extends to the positive characteristic restriction given above. Using a different approach, Basili and Iarrobino proved a much stronger result [1, Theorem 2.16] which reduces to the theorem as stated above. Further still, Iarrobino has pointed out to us that the characteristic zero part follows from a much earlier result of Briançon [3] and the positive characteristic part follows from an earlier result of his own [11, Theorem 2.9].

In this paper, we consider the presence of the strong Lefschetz property for homogeneous artinian quotients of $R=K[x, y]$, where the characteristic of $K$ is positive. In Section 2 we recall some needed definitions and introduce the width function of a monomial ideal. The possible width functions are classified in Proposition 2.6, which is analogous to Macaulay's theorem for Hilbert functions. In Section 3, we derive conditions to determine when the multiplication map $\times \ell^{t}$ : $[R / I]_{d} \rightarrow[R / I]_{d+t}$ has maximal rank for monomial quotients of $R$. 
Section 4 contains the main results of this paper. In particular, Theorem 4.3 bounds the characteristics in which the strong Lefschetz property can be absent from monomial quotients by means of the width function. From this, we recover Theorem 1.2 using different techniques than used in $[\mathbf{1}, \mathbf{9}]$. Furthermore, we classify when the bounds in Theorems 4.3 and 1.2 are sharp in Corollaries 4.7 and 4.8, respectively. In Theorem 4.11, we show that a monomial quotient always has the strong Lefschetz property if and only if it is an artinian quotient of a lexsegment ideal. Further, we provide an infinite family of examples of non-monomial complete intersections that also satisfy this property in Example 4.12.

Throughout the remainder of this paper $R=K[x, y]$, where $K$ is an infinite field of characteristic $p \geq 0$.

2. The width function. Let $I$ be a homogeneous ideal of $S=$ $K\left[x_{1}, \ldots, x_{n}\right]$. Recall that each component $[S / I]_{d}$ is a finite-dimensional $K$-vector space, and the Hilbert function of $S / I$ is the function $h_{S / I}$ : $\mathbf{N}_{0} \rightarrow \mathbf{N}_{0}$, where $h(d):=h_{S / I}(d):=\operatorname{dim}_{K}[S / I]_{d}$. If there is an integer $r$ such that $h(i)>0$ if and only if $0 \leq i \leq r$, then $S / I$ is artinian; in this case, $r$ is the regularity of $S / I$ and is denoted reg $S / I$. If $S / I$ is artinian and $r=\operatorname{reg} S / I$, then we call the finite sequence $(h(0), \ldots, h(r))$, where $h=h_{S / I}$, the $h$-vector of $S / I$. Further still, the initial degree of $I$ is the smallest degree of a minimal generator of $I$ and is denoted indeg $I$. Thus, $[S / I]_{i} \cong[S]_{i}$ for $0 \leq i<\operatorname{indeg} I$.

2.1. Lexsegment ideals and Macaulay's theorem. Suppose $x_{1}>$ $\cdots>x_{n}$ in $S$. The degree lexicographic order on the monomials in $S$ is given by $x_{1}^{a_{1}} \cdots x_{n}^{a_{n}}>x_{1}^{b_{1}} \cdots x_{n}^{b_{n}}$ if either $\sum_{i=1}^{n} a_{i}>$ $\sum_{i=1}^{n} b_{i}$ or $\sum_{i=1}^{n} a_{i}=\sum_{i=1}^{n} b_{i}$ and the leftmost nonzero component of the vector $\left(b_{1}, \ldots, b_{n}\right)-\left(a_{1}, \ldots, a_{n}\right)$ is negative. On the other hand, the degree reverse lexicographic order on the monomials in $S$ is given by $x_{1}^{a_{1}} \cdots x_{n}^{a_{n}}>x_{1}^{b_{1}} \cdots x_{n}^{b_{n}}$ if either $\sum_{i=1}^{n} a_{i}>\sum_{i=1}^{n} b_{i}$ or $\sum_{i=1}^{n} a_{i}=\sum_{i=1}^{n} b_{i}$ and the rightmost nonzero component of the vector $\left(b_{1}, \ldots, b_{n}\right)-\left(a_{1}, \ldots, a_{n}\right)$ is positive. Notice that in the case of two variables $(n=2)$, these two orders are the same.

A monomial ideal $I$ in $S$ is lexsegment in degree $d$ if, for any two monomials $u, v \in[I]_{d}$ and any monomial $m \in[S]_{d}$ such that $u \leq m \leq v$ in the degree lexicographic order, then $m \in I$. If $I$ is lexsegment in 
every degree, then $I$ is a (completely) lexsegment ideal. Further, a lexsegment ideal $I$ is an initial lexsegment ideal if $x_{1}^{d} \in I$ for every degree $d$ such that $[I]_{d} \neq 0$.

In order to state Macaulay's theorem (see, e.g., [10, Theorem 6.3.8]), we must first define some notation. Let $a$ and $d$ be positive integers. The Macaulay expansion of $a$ with respect to $d$ is the unique expansion $a=\left(\begin{array}{c}a_{d} \\ d\end{array}\right)+\cdots+\left(\begin{array}{c}a_{k} \\ k\end{array}\right)$, where $a_{d}>\cdots>a_{k} \geq k \geq 1$. Further, we define $a^{\langle d\rangle}=\left(\begin{array}{c}a_{d}+1 \\ d+1\end{array}\right)+\cdots+\left(\begin{array}{c}a_{k}+1 \\ k+1\end{array}\right)$, and we set $0^{\langle d\rangle}=0$ for all positive integers $d$.

Theorem 2.1 (Macaulay's theorem). Let $h: \mathbf{N}_{0} \rightarrow \mathbf{N}_{0}$ be a function. The following statements are equivalent:

(i) $h$ is the Hilbert function of a standard graded $K$-algebra,

(ii) $h$ is the Hilbert function of an initial lexsegment quotient in $h(1)$ variables, and

(iii) $h(0)=1$ and $h(d+1) \leq h(d)^{\langle d\rangle}$ for all $d \geq 1$.

This allows us to immediately classify the Hilbert functions of ideals in two variables.

Proposition 2.2. Let $h: \mathbf{N}_{0} \rightarrow \mathbf{N}_{0}$ be a function. The function $h$ is the Hilbert function of some (proper) homogeneous quotient in $R=K[x, y]$ if and only if there exists a nonnegative integer $d$ so that $h(j)=j+1$ for $0 \leq j \leq d$ and $h(j) \geq h(j+1) \geq 0$ for all $j \geq d$.

Proof. The Macaulay expansion of $j+1$ with respect to $j$ is $j+1=$ $\left(\begin{array}{c}j+1 \\ j\end{array}\right)$, and so $(j+1)^{\langle j\rangle}=\left(\begin{array}{c}j+2 \\ j+1\end{array}\right)=j+2$. Thus, the Hilbert function of any (proper) homogeneous quotient in $R$ must strictly increase exclusively up to indeg $I$, and further $h_{R / I}(\operatorname{indeg} I) \leq \operatorname{indeg} I$.

If $a$ and $j$ are positive integers so that $a \leq j$, then the Macaulay expansion of $a$ is $a=\left(\begin{array}{l}j \\ j\end{array}\right)+\cdots+\left(\begin{array}{l}j-a+1 \\ j-a+1\end{array}\right)$, and so $a^{\langle j\rangle}=\left(\begin{array}{l}j+1 \\ j+1\end{array}\right)+\cdots+$ $\left(\begin{array}{c}j-a+2 \\ j-a+2\end{array}\right)=a$. Thus, once $h_{R / I}$ is not strictly increasing, it must weakly decrease.

Moreover, we classify the Hilbert functions that force a monomial ideal to be lexsegment. 
Lemma 2.3. Suppose $h: \mathbf{N}_{0} \rightarrow \mathbf{N}_{0}$ is the Hilbert function of some quotient of $R=K[x, y]$. Every monomial quotient $R / I$ with $h_{R / I}=h$ is a lexsegment quotient if and only if, for every nonnegative integer $d$ such that $h(d)>h(d+1)$, we have $h(d+1)=h(d+2)$.

Proof. We prove the negation of the desired statement in two parts. Moreover, all comparisons of monomials in $R=K[x, y]$ are in the degree lexicographic order with $x>y$, and all ordered sets are presented in ascending order.

Suppose that there exists a nonnegative integer $d$ such that $h(d)>$ $h(d+1)$ and $h(d+1) \neq h(d+2)$. By Proposition 2.2, once a Hilbert function is weakly decreasing, then it must remain so; hence, $h(d+1)>h(d+2)$. Let $I$ be the initial lexsegment ideal with Hilbert function $h$, as guaranteed by Macaulay's theorem (see Theorem 2.1). By construction, $[I]_{d}$ is spanned by $a=d+1-h(d)$ monomials of degree $d$, in particular, by the set $A=\left\{x^{d-a+1} y^{a-1}, \ldots, x^{d}\right\}$. Similarly, $[I]_{d+1}$ and $[I]_{d+2}$ are spanned by $b=d+2-h(d+1)$ monomials of degree $d+1$ and $c=d+3-h(d+2)$ monomials of degree $d+2$, respectively; let $B=\left\{x^{d-b+2} y^{b-1}, \ldots, x^{d+1}\right\}$ and $C=\left\{x^{d-c+3} y^{c-1}, \ldots, x^{d+2}\right\}$ be those monomials.

Let $B^{\prime}=\left\{x^{d-b+1} y^{b}, x^{d-b+3} y^{b-2}, \ldots, x^{d+1}\right\}$. Since $h(d)>h(d+1)$, we have that $b-a \geq 2$ and so every product of a member of $A$ with either $x$ or $y$ is in $B^{\prime}$; in particular, $x^{d-a+1} y^{a-1} \cdot y$ is in $B^{\prime}$ since $a \leq b-2$. Further, since $h(d+1)>h(d+2)$, we have that $c-b \geq 2$. Hence, every product of a member of $B^{\prime}$ with either $x$ or $y$ is in $C$; in particular, $x^{d-b+1} y^{b} \cdot y$ is in $C$ since $b+1 \leq c-1$. Let $J$ be given by $[J]_{i}=[I]_{i}$ for $i \neq d+1$, and suppose $[J]_{d+1}$ is spanned by $B^{\prime}$. So $J$ is an ideal with Hilbert function $h_{R / J}=h$ but is not lexsegment in degree $d+1$.

Now suppose that there exists a monomial quotient $R / I$ with $h_{R / I}=$ $h$ that is not lexsegment. That is, there is a degree, say, $d+1$, such that $[I]_{d+1}$ is spanned by $d+2-h(d+1)$ monomials where there are at most $d-h(d+1)$ pairs of consecutive monomials. For every monomial $m$ in $[I]_{d},\{y m, x m\}$ forms a consecutive pair in $[I]_{d+1}$, so there can be at most $d-h(d+1)$ monomials spanning $[I]_{d}$. Since exactly $d+1-h(d)$ monomials span $[I]_{d}$, then $h(d+1)+1 \leq h(d)$. Moreover, for every monomial $\mathrm{m}^{\prime}$ in $[I]_{d+1}$, the monomials $\mathrm{xm}^{\prime}$ and $\mathrm{ym}^{\prime}$ are in $[I]_{d+2}$. However, consecutive monomials overlap in exactly one multiple. Hence, there are at least $2(d+2-h(d+1))-(d-h(d+1))=$ 
$d+4-h(d+1)$ monomials in $[I]_{d+2}$. Since exactly $d+3-h(d+2)$ monomials span $[I]_{d+2}$, then $h(d+2)+1 \leq h(d+1)$. Thus, we have that $h(d)>h(d+1)>h(d+2)$.

2.2. The width function of a monomial ideal. Throughout this section, all comparisons of monomials in $R=K[x, y]$ are in the degree lexicographic order with $x>y$.

Let $I$ be a (not necessarily artinian or lexsegment) monomial ideal of $R$. The width function of $R / I$ is the function $w_{R / I}: \mathbf{N}_{0} \rightarrow \mathbf{N}_{0}$ defined as follows. If $0 \leq d<\operatorname{indeg} I$, then $w_{R / I}(d)=0$. Suppose $d \geq \operatorname{indeg} I$. Let $b$ be the smallest integer so that $x^{b} y^{d-b} \in I$, and let $c$ be the largest integer so that $x^{c} y^{d-c} \in I$. Hence, $w_{R / I}(d)=c-b+1$ is the "width" of the monomials in $[I]_{d}$. If $R / I$ is artinian and $r=\operatorname{reg} R / I$, then we call the finite sequence $(w(0), \ldots, w(r))$, where $w=w_{R / I}$, the $w$-vector of $R / I$.

Remark 2.4. For a monomial ideal $I$ of $R$, the number of degree $d$ monomials not in $I$ between the smallest and largest degree $d$ monomials in $I$ is $w_{R / I}(d)+h_{R / I}(d)-(d+1)$, which is zero precisely when $I$ is lexsegment in degree $d$. Thus, this number can be thought of as the "lexsegment defect" of $I$ in degree $d$.

Example 2.5. Let $I=\left(x^{6}, x^{3} y, x y^{4}, y^{5}\right)$. The $h$-vector of $R / I$ is $(1,2,3,4,4,2)$ and the $w$-vector of $R / I$ is $(0,0,0,0,1,5)$. Notice that, in degree 4 , the only monomial in $[I]_{4}$ is $x^{3} y$; hence, $w_{R / I}(4)=1$. However, in degree 5 , the monomials in $[I]_{5}$ are $y^{5}, x y^{4}, x^{3} y^{2}$ and $x^{4} y$, so $w_{R / I}(5)=5$. In particular, the only nonzero lexsegment defect of $I$ is in degree 5 and is 1 , which corresponds to the monomial $x^{2} y^{3}$.

We now classify the possible width functions of monomial ideals.

Proposition 2.6. Let $w: \mathbf{N}_{0} \rightarrow \mathbf{N}_{0}$ be a function, and let $R=K[x, y]$. The following statements are equivalent:

(i) $w=w_{R / I}$, where $I$ is a monomial ideal,

(ii) $w=w_{R / I}$, where $I$ is an initial lexsegment ideal,

(iii) $w(d)=d+1-h_{R / I}(d)$, where $I$ is an initial lexsegment ideal, and 
(iv) either $w(d)=d+1$ for all $d \geq 0$ or there exists an integer $m>0$ so that $w(d)=0$ for $d<m$ and $1 \leq w(d)<w(d+1) \leq d+2$, for $d \geq m$.

Proof. Clearly, (ii) implies (i). We proceed by showing (i) $\Rightarrow$ (iv) $\Rightarrow$ (iii) $\Rightarrow$ (ii).

(i) $\Rightarrow$ (iv). Let $I$ be a monomial ideal with width function $w=w_{R / I}$. If $I=R$, then $w(d)=d+1$ for all $d$. Suppose $I \neq R$; then indeg $I \geq 1$. By construction, $w(d)=0$ for $d<\operatorname{indeg} I$. Let $d \geq \operatorname{indeg} I$, and set $b=\min \left\{i \mid x^{i} y^{d-i} \in I\right\}$ and $c=\max \left\{i \mid x^{i} y^{\bar{d}-i} \in I\right\}$, i.e., $w(d)=c-b+1$. Clearly, $0 \leq b \leq c \leq d$, so $1 \leq w(d) \leq d+1$. Moreover, since $x^{b} y^{d-b}$ and $x^{c} y^{d-c}$ are both in $I$, then $x^{b} y^{d+1-b}$ and $x^{c+1} y^{d-c}$ are both in $I$. Thus, $b \geq \min \left\{i \mid x^{i} y^{d+1-i}\right\}$ and $c+1 \leq \max \left\{i \mid x^{i} y^{d+1-i} \in I\right\}$, and so $w(d+1) \geq c+1-b+1>w(d)$.

(iv) $\Rightarrow$ (iii). If $w(d)=d+1$ for all $d \geq 0$, then $w(d)=d+1-h_{R / I}(d)$, where $I=R$, which is clearly an initial lexsegment ideal. Suppose now $w: \mathbf{N}_{0} \rightarrow \mathbf{N}_{0}$ is a function such that there exists an integer $m>0$ so that $w(d)=0$ for $d<m$ and $1 \leq w(d)<w(d+1) \leq d+2$, for $d \geq m$. Define $h: \mathbf{N}_{0} \rightarrow \mathbf{N}_{0}$ by $h(d)=d+1-w(d)$ for $d \geq 0$. Thus, $h(0)=1, h(1) \leq 2$, and $h(d)=d+1$ for $0 \leq d<m$. Moreover, since $w(d)<w(d+1) \leq d+2$ for $d \geq m$, then $h(d)=$ $d+1-w(d) \geq d+2-w(d+1)=h(d+1) \geq 0$. Hence, by Proposition 2.2, $h$ is a Hilbert function of some proper homogeneous quotient of $R$. Thus, by Macaulay's theorem (see Theorem 2.1), there exists an initial lexsegment ideal $I$ such that $h=h_{R / I}$.

(iii) $\Rightarrow$ (ii). Let $w(d)=d+1-h_{R / I}(d)$, where $I$ is an initial lexsegment ideal. As $I$ is lexsegment in degree $d$, for all $d$, then there are $d+1-h_{R / I}(d)=w(d)$ monomials of degree $d$ in $I$. Moreover, these $w(d)$ monomials are consecutive in the degree lexicographic order and so $w_{R / I}(d)=w(d)$.

Thus, the four statements (i)-(iv) are indeed equivalent.

Further, we classify the width functions that force a monomial ideal to be lexsegment.

Lemma 2.7. Suppose $w: \mathbf{N}_{0} \rightarrow \mathbf{N}_{0}$ is the width function of some monomial quotient of $R=K[x, y]$. Every monomial quotient $R / I$ with 
$w_{R / I}=w$ is lexsegment if and only if, for every nonnegative integer $d$, we have $0 \leq w(d+1)-w(d) \leq 2$.

Proof. We prove the negation of the desired statement in two parts. Moreover, all comparisons of monomials in $R=K[x, y]$ are in the degree lexicographic order with $x>y$, and all ordered sets are presented in ascending order.

Suppose that there exists a nonnegative integer $d$ so that $w(d+1)-$ $w(d)>2$. Let $I$ be the initial lexsegment ideal with width function $w_{R / I}=w$, as guaranteed by Proposition 2.6. Hence, $[I]_{d+1}$ is spanned by the $w(d+1)$ monomials $B=\left\{x^{d-w(d+1)+2} y^{w(d+1)-1}, \ldots, x^{d+1}\right\}$. Notice that the next to last monomial of $B$, namely, $x^{d-w(d+1)+3} y^{w(d+1)-2}$, is not a multiple of a monomial in $[I]_{d}$, since $x^{d-w(d)+1} y^{w(d)-1}$ is the smallest monomial in $[I]_{d}$ and $w(d) \leq w(d+1)-3$.

Let $B^{\prime}$ be $B \backslash\left\{x^{d-w(d)+1} y^{w(d)-1}\right\}$. Hence, if $J$ given by $[J]_{i}=[I]_{i}$ for $i \neq d+1$ and $[J]_{d+1}$ is spanned by $B^{\prime}$, then $J$ is an ideal with width function $w_{R / J}=w$, and $J$ is not lexsegment in degree $d+1$.

Now suppose that there exists a monomial quotient $R / I$ with $w_{R / I}=$ $w$ that is lexsegment in every degree $i \leq d$ but not lexsegment in degree $d+1$. Thus, there are $w(i)$ consecutive monomials in $I$ of degree $i$ for $i \leq d$, and there are less than $w(d+1)$ monomials in $I$ of degree $d+1$. By Proposition 2.6, we have $w(d)<w(d+1)$. Since $I$ is not lexsegment in degree $d+1$, then there must be at least one monomial that is not consecutive to one of the $w(d)+1$ consecutive multiples of the $w(d)$ consecutive monomials in degree $d$. Thus the width in degree $d+1$ must be at least two larger than $w(d)+1$ (one for the absent monomial and one for the guaranteed non-consecutive monomial). That is, $w(d+1) \geq w(d)+3$.

3. Maximal rank multiplication maps. Throughout this section, all comparisons of monomials in $R=K[x, y]$ are in the degree lexicographic order with $x>y$, and all ordered sets are presented in ascending order.

Proposition 3.1. [15, Proposition 2.2]. Let I be a monomial artinian ideal in the ring $S=K\left[x_{1}, \ldots, x_{n}\right]$. The quotient $S / I$ has the weak 
(strong) Lefschetz property if and only if $x_{1}+\cdots+x_{n}$ is a weak (strong) Lefschetz element of $S / I$.

Hence, Theorem 1.1 implies that we need only look at the maps between equi-dimensional components.

Lemma 3.2. Let $I$ be a monomial artinian ideal in $R$. The quotient $R / I$ has the strong Lefschetz property if and only if the map $\times(x+y)^{t}$ : $[R / I]_{d} \rightarrow[R / I]_{d+t}$ is a bijection for all integers $d \geq 0$ and $t \geq 1$ where $h_{R / I}(d)=h_{R / I}(d+t)=d+1$.

Proof. The presence of the strong Lefschetz property clearly implies the second condition.

Suppose now that the second condition holds. Let $\varphi_{d, d+t}$ be the map $\times(x+y)^{t}:[R / I]_{d} \rightarrow[R / I]_{d+t}$, and so $\varphi_{a, a+b+c}=\varphi_{a+b, a+b+c} \circ \varphi_{a, a+b}$.

Theorem 1.1 implies that $\varphi_{d, d+1}$ always has maximal rank. In particular, $\varphi_{d, d+1}$ is injective for $0 \leq d<\operatorname{indeg} I$ and surjective for indeg $I \leq d$. Since $\varphi_{d, d+t}=\varphi_{d+t-1, d+t} \circ \cdots \circ \varphi_{d, d+1}$, if $d+t \leq$ indeg $I$ (respectively, $d>\operatorname{indeg} I$ ), then each term in the composition is injective (respectively, surjective) and so $\varphi_{d, d+t}$ is injective (respectively, surjective).

Now suppose that $d<\operatorname{indeg} I<d+t$. Since $d+t>$ indeg $I$, then $h(d+t)-1 \leq \operatorname{indeg} I$, and so $h(h(d+t)-1)=h(d+t)$ by Proposition 2.2. Thus, by assumption, $\varphi_{h(d+t)-1, d+t}$ is a bijection. If $d<h(d+t)-1$, then $\varphi_{d, d+t}=\varphi_{h(d+t)-1, d+t} \circ \varphi_{d, h(d+t)-1}$. Since $h(d+t)-1 \leq \operatorname{indeg} I$, $\varphi_{d, h(d+t)-1}$ is injective. Hence, $\varphi_{d, d+t}$ is injective as the composition of injective functions is injective. On the other hand, if $d>h(d+t)-1$, then $\varphi_{h(d+t)-1, d+t}=\varphi_{d, d+t} \circ \varphi_{h(d+t)-1, d}$. Hence, $\varphi_{d, d+t}$ is surjective as $\varphi_{h(d+t)-1, d+t}$ is, by assumption, a bijection.

Let $I$ be a monomial ideal in $R$, and let $d \geq 0$ and $t \geq 1$ be any integers such that $h(d)=h(d+t)=d+1$. Suppose the ordered monomials $\left\{x^{b_{0}} y^{d+t-b_{0}}, \ldots, x^{b_{d}} y^{d+t-b_{d}}\right\}$ span $[R / I]_{d+t}$. Note that the ordering implies that $0 \leq b_{0}<\cdots<b_{d} \leq d+t$. The map $\times(x+y)^{t}:[R / I]_{d} \rightarrow[R / I]_{d+t}$ is given by the $(d+1) \times(d+1)$ matrix $N_{R / I}(d, d+t)$, where the entry $(i, j)$ is the coefficient on $x^{b_{j}} y^{d+t-b_{j}}$ in $x^{i} y^{d-i}(x+y)^{t}$, i.e., $\left(\begin{array}{c}t \\ b_{j}-i\end{array}\right)$. 
Example 3.3. Let $I=\left(x^{10}, y^{7}\right)$. Hence, $h_{R / I}(5)=h_{R / I}(10)=6$, and thus $N_{R / I}(5,5)$ is the $6 \times 6$ matrix

$$
\left(\begin{array}{cccccc}
5 & 10 & 10 & 5 & 1 & 0 \\
1 & 5 & 10 & 10 & 5 & 1 \\
0 & 1 & 5 & 10 & 10 & 5 \\
0 & 0 & 1 & 5 & 10 & 10 \\
0 & 0 & 0 & 1 & 5 & 10 \\
0 & 0 & 0 & 0 & 1 & 5
\end{array}\right) .
$$

The determinant of this matrix is $210=2 \cdot 3 \cdot 5 \cdot 7$, and so the map $\times(x+y)^{5}:[R / I]_{5} \rightarrow[R / I]_{10}$ has maximal rank if and only if char $K=0$ or char $K>7$.

The determinant of $N_{-}(d, d+t)$ can be given by a closed form.

Lemma 3.4. Let $I$ be a monomial ideal in $R$. If $d \geq 0$ and $t \geq 1$ are integers so that $h_{R / I}(d)=h_{R / I}(d+t)=d+1$, then $\left|\operatorname{det} N_{R / I}(d, d+t)\right|$ is

$$
\prod_{0 \leq i<j \leq s-r}\left(b_{r+j}-b_{r+i}\right) \prod_{i=0}^{s-r} \frac{(t+i) !}{\left(t+s-b_{r+i}\right) !\left(b_{r+i}-r\right) !}
$$

where the ordered monomials $\left\{x^{b_{0}} y^{d+t-b_{0}}, \ldots, x^{b_{d}} y^{d+t-b_{d}}\right\}$ span $[R / I]_{d+t}$, $r:=\max \left(\{0\} \cup\left\{k+1 \mid b_{k}=k\right\}\right)$, and $s:=\min \left(\{d\} \cup\left\{k-1 \mid b_{k}=t+k\right\}\right)$.

If $r=s+1$, then the determinant is one; otherwise, the largest factor of the above closed form is $w_{R / I}(d+t)-1$, and it appears exactly once.

Proof. For $0 \leq i<r$, we have $b_{i}=i$, and so the $(i, j)$ entry of $N_{R / I}(d, d+t)$ is $\left(\begin{array}{c}t \\ j-i\end{array}\right)$, i.e., 1 if $i=j$ and 0 if $i>j$. Similarly, for $s<j \leq d$, we have that $b_{j}=t+j$ and so the $(i, j)$ entry of $N_{R / I}(d, d+t)$ is $\left(\begin{array}{c}t \\ t+j-i\end{array}\right)$, i.e., is 1 if $i=j$ and 0 if $i<j$.

Partitioning $N_{R / I}(d, d+t)$ into a block matrix with square diagonal matrices with sizes $r, s-r+1$, and $d-s$, respectively, yields

$$
N_{R / I}(d, d+t)=\left(\begin{array}{ccc}
U & A & 0 \\
0 & \widehat{N} & 0 \\
0 & B & L
\end{array}\right)
$$


where $U$ is a square upper-triangular matrix with ones on the diagonal, $L$ is a square lower-triangular matrix with ones on the diagonal, and $\widehat{N}$ is an $(s-r+1) \times(s-r+1)$ square matrix with entry $(i, j)$ given by $\left(\begin{array}{c}t \\ b_{r+j}-(r+i)\end{array}\right)$. Using the block matrix formula for the determinant (twice), we have

$$
\operatorname{det} N_{R / I}(d, d+t)=\operatorname{det} U \cdot \operatorname{det} \widehat{N} \cdot \operatorname{det} L=\operatorname{det} \widehat{N} .
$$

Set $A:=t, n:=s-r+1$ and $L_{j}:=b_{r+j-1}-r+1$. The determinant evaluation of $\widehat{N}$, hence of $N_{R / I}(d, d+t)$, follows immediately from [5, equation (12.5)]. Re-indexing to start from 0 instead of 1 yields the stated result.

If $r=s+1$, then the products are all empty; hence, the determinant is one. Suppose $r \leq s$. Notice that $b_{s}<t+s$ and $b_{r}>r$. Hence, the largest factor in the first product $b_{s}-b_{r}$ is less than $t+s-r$, and the largest factor in the numerator of the second product is $t+s-r$. The largest factor in the denominator of the second product is the maximum of $t+s-b_{r}$ and $b_{s}-r$, both of which are less than $t+s-r$. Hence, the largest factor of the products in the formula is $t+s-r$.

Notice that, by the definitions of $r$ and $s, x^{r} y^{d+t-r}$ and $x^{t+s} y^{d-s}$ are the degree lexicographically smallest and largest monomials in $[I]_{d+t}$, respectively. Hence, $w_{R / I}(d+t)=t+s-r+1$, and so the largest factor of the products in the formula is $w_{R / I}(d+t)-1$.

Remark 3.5. Let $I$ be a monomial artinian ideal in $R=K[x, y]$. If $N_{R / I}(d, d+t)$ is square, as in the preceding lemma, then the determinant can be interpreted combinatorially. In particular, using a beautiful theorem first given by Lindström [13, Lemma 1] and stated independently by Gessel and Viennot [8, Theorem 1], the determinant can be interpreted as the enumeration of families of nonintersecting lattice paths on a finite sublattice of $\mathcal{Z}^{2}$. This connection follows from work of the author with Nagel [7], as the presence of the strong Lefschetz property of $R / I$ is reflected in the presence of the weak Lefschetz property of $R[z] /\left(I+\left(z^{t}\right)\right)$. (The connection between the strong Lefschetz property in $n$ variables and the weak Lefschetz property in $n+1$ variables has been used more generally; see, e.g., $[4,6,14,16]$.) 
The matrix $\widehat{N}$ in the proof of Lemma 3.4 is the matrix $N_{R / J}(s-$ $r, s-r+t)$, where $J$ is the ideal $\left(x^{b_{r}-r} y^{t+s-b_{r}}, \ldots, x^{b_{s}-r} y^{t+s-b_{s}}\right)+$ $(x, y)^{t+s-r+1}$.

Example 3.6. Let $I=\left(x^{15}, x^{10} y^{2}, x^{2} y^{9}, y^{15}\right)$. Hence, $h_{R / I}(9)=10=$ $h_{R / I}(14)$, so $N_{R / I}(9,14)$ is the $10 \times 10$ matrix

$$
\left(\begin{array}{cc|cccccc|cc}
1 & 5 & 10 & 5 & 1 & 0 & 0 & 0 & 0 & 0 \\
0 & 1 & 10 & 10 & 5 & 1 & 0 & 0 & 0 & 0 \\
\hline 0 & 0 & 5 & 10 & 10 & 5 & 1 & 0 & 0 & 0 \\
0 & 0 & 1 & 5 & 10 & 10 & 5 & 1 & 0 & 0 \\
0 & 0 & 0 & 1 & 5 & 10 & 10 & 5 & 0 & 0 \\
0 & 0 & 0 & 0 & 1 & 5 & 10 & 10 & 0 & 0 \\
0 & 0 & 0 & 0 & 0 & 1 & 5 & 10 & 0 & 0 \\
0 & 0 & 0 & 0 & 0 & 0 & 1 & 5 & 0 & 0 \\
\hline 0 & 0 & 0 & 0 & 0 & 0 & 0 & 1 & 1 & 0 \\
0 & 0 & 0 & 0 & 0 & 0 & 0 & 0 & 5 & 1
\end{array}\right) .
$$

Notice that the upper-left $2 \times 2$ matrix is upper-triangular, the lowerleft $2 \times 2$ matrix is lower-triangular, and the central $6 \times 6$ matrix has determinant $210=2 \cdot 3 \cdot 5 \cdot 7$. Thus, the determinant of $N_{R / I}(9,14)$ has magnitude 210 , and so the map $\times(x+y)^{5}:[R / I]_{9} \rightarrow[R / I]_{14}$ has maximal rank if and only if char $K=0$ or char $K>7$.

Indeed, the central $6 \times 6$ matrix is the same as the matrix in Example 3.3 and is the matrix $\widehat{N}$ in the proof of Lemma 3.4. Furthermore, following the preceding remark, we note that $d=9, t=5, r=2$ and $s=7$ for $R / I$ in degree 14. Hence, $\widehat{N}$ is the matrix $N_{R / J}(s-r, s-r+t)=$ $N_{R / J}(5,10)$, where $J=\left(x^{10}, x^{3} y^{7}, x^{2} y^{8}, x y^{9}, y^{10}\right)$. Notice that $R / J$ and the quotient considered in Example 3.3 are the same in degrees 5 and 10 .

Analyzing the formula in Lemma 3.4, we determine exactly when $|\operatorname{det} N|=1$.

Corollary 3.7. Let $I$ be a monomial ideal in $R$, and let $d \geq 0$ and $t \geq 1$ be integers so that $h_{R / I}(d)=h_{R / I}(d+t)=d+1$. The following statements are equivalent:

(i) $\left|\operatorname{det} N_{R / I}(d, d+t)\right|=1$, 
(ii) $w_{R / I}(d+t)=t$, and

(iii) $I$ is lexsegment in degree $d+t$.

Specifically, if $t=1$, then $\left|\operatorname{det} N_{R / I}(d, d+t)\right|=1$.

Proof. By Remark 2.4, $w_{R / I}(d+t)=d+t-1-h_{R / I}(d+t)=t$ if and only if $I$ is lexsegment in degree $d$, hence (ii) is equivalent to (iii).

Suppose $[R / I]_{d+t}$ is spanned by the ordered monomials $\left\{x^{b_{0}} y^{d+t-b_{0}}\right.$, $\left.\ldots, x^{b_{d}} y^{d+t-b_{d}}\right\}$. Set $r:=\max \left(\{0\} \cup\left\{k+1 \mid b_{k}=k\right\}\right)$ and $s:=$ $\min \left(\{d\} \cup\left\{k-1 \mid b_{k}=t+k\right\}\right)$. Thus, $r<b_{r}<\cdots<b_{s}<s+t$. By Lemma $3.4,\left|\operatorname{det} N_{R / I}(d, d+t)\right|$ is

$$
\prod_{0 \leq i<j \leq s-r}\left(b_{r+j}-b_{r+i}\right) \prod_{i=0}^{s-r} \frac{(t+i) !}{\left(t+s-b_{r+i}\right) !\left(b_{r+i}-r\right) !} .
$$

Further, notice that $w_{R / I}(d+t)=s-r+t+1$.

We prove (i) is equivalent to (ii) via an induction on $t$.

Step 1: Setup. Let $r, s$, and $t$ be integers such that $0 \leq r \leq s+1$ and $t \geq 1$. Let $b_{r}, \ldots, b_{s}$ be integers such that $r<b_{r}<\cdots<b_{s}<s+t$. Define $D\left(r, s, t,\left\{b_{r}, \ldots, b_{s}\right\}\right)$ to be

$$
\prod_{0 \leq i<j \leq s-r}\left(b_{r+j}-b_{r+i}\right) \prod_{i=0}^{s-r} \frac{(t+i) !}{\left(t+s-b_{r+i}\right) !\left(b_{r+i}-r\right) !} .
$$

Clearly, $D\left(r, s, t,\left\{b_{r}, \ldots, b_{s}\right\}\right) \geq 1$ for all valid arguments. Moreover, if $r=s+1$, then $D(s+1, s, t, \emptyset)=1$ for all $s$ and $t$, as the products are all empty.

Step 2: Base case. Suppose $t=1$. If $r \leq s$, then $r<b_{r}<\cdots<$ $b_{s}<s+1$, which implies there are at least $s-r+1$ distinct integers exclusively between $r$ and $s+1$; however, there are only $s-r$ such integers. Hence, $r=s+1$, and so $D(s+1, s, 1, \emptyset)=1$ for every $s$. (We also note that this follows immediately from Theorem 1.1.)

Step 3: Induction step. Suppose $t \geq 2$. If $r=s$, then $D\left(r, r, t,\left\{b_{r}\right\}\right)=\left(\begin{array}{c}t \\ b_{r}-r\end{array}\right) \geq t$, as $1 \leq b_{r}-r \leq t-1$. Hence we may assume $r<s$. We consider two cases.

Case (a). When $b_{s}<s+t-1$. Clearly, $\left(r, s, t+1,\left\{b_{r}, \ldots, b_{s}\right\}\right)$ forms a valid argument for $D(\cdot)$. Indeed, we can rewrite $D(r, s, t+$ 
$\left.1,\left\{b_{r}, \ldots, b_{s}\right\}\right)$ in terms of $D\left(r, s, t,\left\{b_{r}, \ldots, b_{s}\right\}\right)$ as follows:

$$
\begin{aligned}
D\left(r, s, t+1,\left\{b_{r}, \ldots, b_{s}\right\}\right)= & \prod_{0 \leq i<j \leq s-r}\left(b_{r+j}-b_{r+i}\right) \\
& \times \prod_{i=0}^{s-r} \frac{((t+1)+i) !}{\left((t+1)+s-b_{r+i}\right) !\left(b_{r+i}-r\right) !} \\
& =\prod_{i=0}^{s-r} \frac{((t+1)+i)}{\left((t+1)+s-b_{r+i}\right)} D\left(r, s, t,\left\{b_{r}, \ldots, b_{s}\right\}\right) .
\end{aligned}
$$

For $0 \leq i \leq s-r$, we have $b_{r+i}>r+i$, and so $(t+1)+s-b_{r+i} \leq$ $t+s+1-(r+i+1)=t+s-r-i$. Further still, $t+1+i=t+s-r-j+1$, where $j=s-r-i$, i.e., $0 \leq j \leq s-r$. Thus, we have

$$
\begin{aligned}
\prod_{i=0}^{s-r}((t+1)+i) & =\prod_{j=0}^{s-r}(t+s-r-j+1) \\
& >\prod_{j=0}^{s-r}(t+s-r-j) \\
& \geq \prod_{i=0}^{s-r}\left((t+1)+s-b_{r+i}\right) .
\end{aligned}
$$

Hence,

$$
\prod_{i=0}^{s-r} \frac{((t+1)+i)}{\left((t+1)+s-b_{r+i}\right)}>1
$$

and $D\left(r, s, t+1,\left\{b_{r}, \ldots, b_{s}\right\}\right)>D\left(r, s, t,\left\{b_{r}, \ldots, b_{s}\right\}\right) \geq 1$.

Case (b): When $b_{s}=s+t-1$. Since $r \leq s$, we have $r \leq$ $(s-1)+1$, and so $\left(r, s-1, t,\left\{b_{r}, \ldots, b_{s-1}\right\}\right)$ forms a valid argument for $D(\cdot)$. Indeed, we can rewrite $D\left(r, s, t,\left\{b_{r}, \ldots, b_{s}\right\}\right)$ in terms of $D\left(r, s-1, t,\left\{b_{r}, \ldots, b_{s-1}\right\}\right)$ as follows:

$$
\begin{aligned}
D & \left(r, s, t,\left\{b_{r}, \ldots, b_{s}\right\}\right) \\
& =\prod_{0 \leq i<j \leq s-r}\left(b_{r+j}-b_{r+i}\right) \prod_{i=0}^{s-r} \frac{(t+i) !}{\left(t+s-b_{r+i}\right) !\left(b_{r+i}-r\right) !} \\
& =\left(\frac{(t+s-r) !}{\left(t+s-b_{s}\right) !\left(b_{s}-r\right) !} \prod_{0 \leq i<s-r} \frac{b_{s}-b_{r+i}}{t+s-b_{r+i}}\right)
\end{aligned}
$$




$$
\begin{aligned}
& \times D\left(r, s-1, t,\left\{b_{r}, \ldots, b_{s-1}\right\}\right) \\
= & \left((t+s-r) \prod_{0 \leq i<s-r} \frac{t+s-1-b_{r+i}}{t+s-b_{r+i}}\right) \\
& \times D\left(r, s-1, t,\left\{b_{r}, \ldots, b_{s-1}\right\}\right) \\
= & \frac{(t+s-r)\left(t+s-1-b_{r}\right)}{t+s-b_{s-1}} D\left(r, s-1, t,\left\{b_{r}, \ldots, b_{s-1}\right\}\right) .
\end{aligned}
$$

Since $r<b_{s-1}$, we have $t+s-r>t+s-b_{s-1}$, and so

$$
\frac{t+s-r}{t+s-b_{s-1}}>1 \text {. }
$$

Thus, $D\left(r, s, t,\left\{b_{r}, \ldots, b_{s}\right\}\right)>D\left(r, s-1, t,\left\{b_{r}, \ldots, b_{s-1}\right\}\right) \geq 1$.

Conclusion. We thus see that $D\left(r, s, t,\left\{b_{r}, \ldots, b_{s}\right\}\right)=1$ if and only if $r=s+1$. This is equivalent to $w_{R / I}(d+t)=t$, as $w_{R / I}(d+t)=$ $s-r+t+1$.

4. The strong Lefschetz property. Let $I$ be a homogeneous ideal of $S=K\left[x_{1}, \ldots, x_{n}\right]$, and fix a term order $<$ on the monomials of $S$ (e.g., the degree lexicographic order). There exists a Zariski open subset $U$ of $\mathrm{GL}_{n}(K)$ such that $\operatorname{in}_{<}(g \cdot I)=$ in $_{<}(h \cdot I)$ for all $g, h \in U$; the ideal $\operatorname{in}_{<}(g \cdot I)$ is the generic initial ideal of $I$ with respect to $<$ and is denoted by $\operatorname{gin} I=\operatorname{gin}_{<} I$.

Remark 4.1. The generic initial ideal is known to be strongly stable if the characteristic of $K$ is zero (see, e.g., [10, Proposition 4.2.6]). For ideals in $K[x, y]$, being strongly stable is equivalent to being lexsegment. However, the generic initial ideal is not so well-behaved in positive characteristic. Indeed, [10, Example 4.2.8] shows that, for every prime $p>0, \operatorname{gin}\left(x^{p}, y^{p}\right)=\left(x^{p}, y^{p}\right)$ if the characteristic of $K$ is $p$.

Proposition 4.2. [19, Proposition 2.8] Let $I$ be a homogeneous artinian ideal in $S=K\left[x_{1}, \ldots, x_{n}\right]$, and let $J$ be the generic initial ideal of I with respect to the degree reverse lexicographic order. The quotient $S / I$ has the weak (strong) Lefschetz property if and only if $S / J$ has the weak (strong) Lefschetz property.

Further, for $R=K[x, y]$ the degree lexicographic and degree reverse 
lexicographic orders are identical. In some cases, we can use the above result to lift statements about monomial ideals to statements about homogeneous ideals.

\subsection{Bounds on the absence of the strong Lefschetz property.} We first use the width function to bound the characteristics in which the strong Lefschetz property can be absent. See Corollary 4.7 for a classification of when this bound is sharp.

Theorem 4.3. Let $I$ be a monomial artinian ideal in $R=K[x, y]$, where $K$ is a field of characteristic $p>0$. The quotient $R / I$ has the strong Lefschetz property if $p \geq w_{R / I}(\operatorname{reg} R / I)$.

Proof. Let $d \geq 0$ and $t \geq 1$ be integers such that $h(d)=h(d+t)=$ $d+1$. The largest factor of the determinant of $N_{R / I}(d, d+t)$ is $w_{R / I}(d+t)-1$ by Lemma 3.4. Hence, $N_{R / I}(d, d+t)$ has maximal rank if $p \geq w_{R / I}(d+t)$.

Further, we have that $w_{R / I}(\operatorname{reg} R / I) \geq w_{R / I}(i)$ for all $0 \leq i \leq$ $\operatorname{reg} R / I$ by Proposition 2.6. This implies that every matrix $N_{R / I}(d, d+$ $t)$ has maximal rank if $p \geq w_{R / I}(\operatorname{reg} R / I)$. Therefore, $R / I$ has the strong Lefschetz property by Lemma 3.2 if $p \geq w_{R / I}(\operatorname{reg} R / I)$.

We now recover Theorem 1.2 with different techniques than those used in $[\mathbf{1}, \mathbf{9}]$. (Nota bene: We have not assumed Theorem 1.2 up to this point; doing so offers no benefit as our approach and desired results are different.) In particular, by weakening the bound in the preceding proof we can generalize from monomial ideals to homogeneous ideals. See Corollary 4.8 for a classification of when this bound is sharp.

Theorem 4.4. Let $I$ be a homogeneous artinian ideal in $R=K[x, y]$, where $K$ is a field of characteristic $p>0$. The quotient $R / I$ has the strong Lefschetz property if $p>\operatorname{reg} R / I$.

Proof. Let $J=\operatorname{gin} I$ under the degree (reverse) lexicographic order. By Theorem 4.3, $R / J$ has the strong Lefschetz property if $p \geq w_{R / J}(\operatorname{reg} R / J)$. By Proposition 2.6 we have that $w_{R / J}(\operatorname{reg} R / J) \leq$ $\operatorname{reg} R / J+1$, and since the generic initial ideal preserves the Hilbert function we have $\operatorname{reg} R / J=\operatorname{reg} R / I$ as $J$ is artinian. Hence, $R / I$ has the strong Lefschetz property if $p>\operatorname{reg} R / I$ by Proposition 4.2. 
Remark 4.5. The bound in the preceding theorem sometimes holds in higher codimension. Let $I$ be a monomial complete intersection in $S=K\left[x_{1}, \ldots, x_{n}\right]$, i.e., $I=\left(x_{1}^{d_{1}}, \ldots, x_{n}^{d_{n}}\right)$. Thus, we have that $S / I$ has the strong Lefschetz property if $p>\operatorname{reg} S / I$ [6, Theorem 3.6(ii)]. Moreover, this bound is sharp in some cases; in particular, if $p=$ $\operatorname{reg} S / I$, then $S / I$ fails to have the strong Lefschetz property [6, Theorem 3.6(i)]. See Corollary 4.8 for a similar statement about the sharpness of the bound in the preceding corollary.

On the other hand, the bound is not true in general, even as a bound for the failure of the weak Lefschetz property. Let $I=$ $\left(x^{20}, y^{20}, z^{20}, x^{3} y^{8} z^{13}\right)$ be an ideal of $S=K[x, y, z]$. In this case, $\operatorname{reg} S / I=50$, and $S / I$ has the weak Lefschetz property if and only if the characteristic of $K$ is not one of the following primes: 2, 3, 5, $7,11,17,19,23$, or 20554657 . This example comes from [7], wherein the presence and absence of the weak Lefschetz property for monomial ideals in codimension three is considered.

We now consider for which characteristics the strong Lefschetz property is absent.

Lemma 4.6. Let $I$ be a monomial artinian ideal in $R=K[x, y]$. If there exists a $j$ such that indeg $I \leq j \leq \operatorname{reg} R / I, w_{R / I}(j) \neq$ $j+1-h_{R / I}(j)$, and $w_{R / I}(j)-1=$ char $K$ is prime, then $R / I$ fails to have the strong Lefschetz property.

Proof. Let $d=h_{R / I}(j)-1$ and $t=j-d$. Then $h_{R / I}(d)=d+1=$ $h_{R / I}(d+t)$. By Lemma 3.4, since $w_{R / I}(d+t)>d+1-h_{R / I}(d+t)$, we have that the largest factor of the formula giving the determinant of $N_{R / I}(d, d+t)$ is $w_{R / I}(d+t)-1=\operatorname{char} K$. This implies that the map $\times(x+y)^{t}:[R / I]_{d} \rightarrow[R / I]_{d+t}$ fails to have maximal rank, i.e., $R / I$ fails to have the strong Lefschetz property.

Using this, we classify exactly when the bound in Theorem 4.3 is sharp.

Corollary 4.7. Let $I$ be a monomial artinian ideal in $R=K[x, y]$, and suppose $p=w_{R / I}(\operatorname{reg} R / I)-1$ is prime. The quotient $R / I$ fails 
to have the strong Lefschetz property in characteristic $p$ if and only if $x^{\operatorname{reg} R / I}, y^{\operatorname{reg} R / I} \in I$.

Proof. Suppose $p=w_{R / I}(\operatorname{reg} R / I)-1$ is prime. If one of $x^{\operatorname{reg} R / I}$ and $y^{\operatorname{reg} R / I}$ is not in $I$, then $R / I$ can only fail to have the strong Lefschetz property in characteristics smaller than $p=w_{R / I}(\operatorname{reg} R / I)-1$ by Theorem 4.3 .

On the other hand, suppose $x^{\operatorname{reg} R / I}, y^{\operatorname{reg} R / I} \in I$. So $w_{R / I}(\operatorname{reg} R / I)$ $\neq \operatorname{reg} R / I+1-h_{R / I}(\operatorname{reg} R / I)$, and hence $R / I$ fails to have the strong Lefschetz property by Lemma 4.6.

As with Theorems 4.3 and 1.2, the above pair of results can be extended to homogeneous ideals, if we strengthen the restrictions on the ideals. This in turn classifies exactly when the bound in Theorem 1.2 is sharp.

Corollary 4.8. Let $I$ be a homogeneous artinian ideal in $R=K[x, y]$, where $K$ is a field of characteristic $p>0$. If $p \leq \operatorname{reg} R / I$ and $x^{p}, y^{p} \in \operatorname{gin} I$ (under the degree lexicographic order), then $R / I$ fails to have the strong Lefschetz property.

In particular, if $\operatorname{reg} R / I$ is prime and $p=\operatorname{reg} R / I$, then $R / I$ fails to have the strong Lefschetz property if and only if $x^{p}, y^{p} \in \operatorname{gin} I$ (under the degree lexicographic order).

Proof. Let $J=$ gin $I$ under the degree (reverse) lexicographic order. If $x^{p}, y^{p} \in J$, then $w_{R / J}(p)=p+1$. Since $p \leq \operatorname{reg} R / I=\operatorname{reg} R / J$, then $w_{R / J}(p) \neq p+1-h_{R / J}(p)$. Hence, by Lemma 4.6, $R / J$ fails to have the strong Lefschetz property, and so $R / I$ fails to have the strong Lefschetz property by Proposition 4.2.

However, the bounds can be far from sharp in some cases.

Example 4.9. Ignoring lexsegment ideals (which always have the strong Lefschetz property by Theorem 4.11), the bounds in Theorems 4.3 and 1.2 are far from sharp in some cases. In particular, consider the ideal $I_{n}=\left(x^{2^{n}}, y^{2}\right)$, where $n \geq 1$. By [6, Lemma 4.2(i)], we have that $I_{n}$ has the strong Lefschetz property if and only if the characteristic of $K$ divides $2^{n}$, i.e., char $K=2$. However, $\operatorname{reg} R / I_{n}=2^{n}$, 
so the regularity bound is sharp if and only if $n=1$. Moreover, $w_{R / I_{n}}\left(\operatorname{reg} R / I_{n}\right)=2^{n}+1$, so the width bound is never sharp for this family.

Remark 4.10. Corollary 4.8 further implies that the maximal degree of a minimal generator is not a good bound. For example, let $I_{p}=$ $\left(x^{(p+1) / 2}, y^{(p+3) / 2}\right)$, where $p$ is an odd prime. Hence, $\operatorname{reg} R / I_{p}=p$ and $x^{p}, y^{p} \in I_{p}$, so by Corollary $4.8 R / I_{p}$ fails to have the strong Lefschetz property in characteristic $p$. Notice that the maximal generating degree of a minimal generator of $I_{p}$ is $(p+3) / 2$, which is less than $p$ for $p>3$.

4.2. Forcing the presence of the strong Lefschetz property. Let $S=K\left[x_{1}, \ldots, x_{n}\right]$ be a polynomial ring, where $K$ is a field of characteristic zero. Migliore and Zanello [18, Theorem 5] classified the Hilbert functions of artinian quotients of $S$ that force the weak Lefschetz property to hold. Similarly, Zanello and Zylinski [20, Corollary 3.3] proved all artinian quotients of $S$ with a given Hilbert function have the strong Lefschetz property if and only if the initial lexsegment ideal with the given Hilbert function has the strong Lefschetz property.

In some cases, the strong Lefschetz property always holds, regardless of characteristic. The following theorem classifies the monomial ideals with this property.

Theorem 4.11. Let $I$ be a monomial artinian ideal in $R=K[x, y]$. The quotient $R / I$ always has the strong Lefschetz property, regardless of the characteristic of $K$, if and only if $I$ is a lexsegment ideal.

Proof. Suppose $I$ is not a lexsegment ideal. There is a degree $j \geq$ indeg $I$ such that $I$ is not lexsegment in degree $j$. Let $d=h_{R / I}(j)-1$ and $t=j-d$. Then $h_{R / I}(d)=d+1=h_{R / I}(d+t)$. By Corollary 3.7, we see that $N_{R / I}(d, d+t)$ does not always have maximal rank; hence, $R / I$ does not always have the strong Lefschetz property.

Suppose now that $I$ is a lexsegment ideal. By Corollary 3.7, the matrices $N_{R / I}(d, d+t)$, where $h(d)=h(d+t)=d+1$, always have maximal rank. Therefore, $R / I$ always has the strong Lefschetz property, regardless of the characteristic of $K$, by Lemma 3.2.

We also see that non-monomial ideals can satisfy this property. 
Example 4.12. Let $R=K[x, y]$, and let $I$ be a homogeneous ideal of $R$. Weibe showed [19, Proposition 2.9] that $R / I$ has the strong Lefschetz property if $R /$ in $I$ does.

Consider the following ideals of $R$.

(i) Let $I_{b}=\left(x^{2}, x y^{b-1}+y^{b}\right)$, where $b \geq 2$. Thus, in $I_{b}=$ $\left(x^{2}, x y^{b-1}, y^{b+1}\right)$.

(ii) Let $J_{b}=\left(x^{3}, x^{2} y^{b-2}+y^{b}\right)$, where $b \geq 3$. Thus, in $J_{b}=$ $\left(x^{3}, x^{2} y^{b-2}, x y^{b}, y^{b+2}\right)$.

In either case, the initial ideal is a lexsegment ideal and so its quotient always has the strong Lefschetz property by the preceding theorem.

We note that $I_{b}$ is a complete intersection of type $(2, b)$, and $J_{b}$ is a complete intersection of type $(3, b)$. In [6, Lemma 4.2], it was shown that the monomial complete intersections of these types fail to have the strong Lefschetz property for some positive characteristic. In particular, the complete intersection type of an ideal of $R$ is insufficient to classify the presence of the strong Lefschetz property in positive characteristic.

Theorem 4.11 implies that no Hilbert function (see Proposition 2.2) or width function (see Proposition 2.6) can force the strong Lefschetz property to be absent in some characteristic for all ideals with the given Hilbert or width function. On the other hand, we can describe a large class of Hilbert functions and width functions that force the strong Lefschetz property to be present.

Using Lemma 2.3, we classify the Hilbert functions that force monomial ideals to always have the strong Lefschetz property, regardless of characteristic.

Proposition 4.13. Suppose $h: \mathbf{N}_{0} \rightarrow \mathbf{N}_{0}$ is the Hilbert function of some homogeneous artinian quotient of $R=K[x, y]$. Every monomial quotient of $R$ such that $h_{R / I}=h$ has the strong Lefschetz property, regardless of the characteristic of $K$, if and only if for every nonnegative integer $d$ such that $h(d)>h(d+1)$ then $h(d+1)=h(d+2)$.

Proof. Combine Lemma 2.3 and Theorem 4.11. 
Hence, we can force homogeneous ideals with these Hilbert functions to have the strong Lefschetz property.

Corollary 4.14. Suppose $h: \mathbf{N}_{0} \rightarrow \mathbf{N}_{0}$ is the Hilbert function of some homogeneous artinian quotient of $R=K[x, y]$. If, for every nonnegative integer $d$ such that $h(d)>h(d+1)$, we have $h(d+1)=$ $h(d+2)$, then every homogeneous artinian quotient $R / I$ with $h_{R / I}=h$ has the strong Lefschetz property.

Proof. Let $R / I$ be some homogeneous artinian quotient with $h_{R / I}=$ $h$. Since gin $I$ preserves the Hilbert function, we have that $h_{R / \operatorname{gin} I}=h$. By Proposition $4.13, R / \operatorname{gin} I$ has the strong Lefschetz property, and so $R / I$ has the strong Lefschetz property by Proposition 4.2 .

Remark 4.15. We must be careful with the ring in which we consider an ideal to be. For example, the ideal $I=\left(x^{2}+y^{2}, x^{3}+y^{3}\right)$ is in $R=K[x, y]$, regardless of the field $K$. However, $I$ is not artinian in a field of characteristic two. Indeed, if char $K=2$, then $(x, y)=(1,1)$ is a non-trivial common zero of the generators of $I$. However, in all other characteristics $R / I$ is artinian and has $h$-vector $(1,2,2,1)$.

Suppose char $K \neq 2$. The reduced Gröbner basis for $I$ is $\left(x^{2}+\right.$ $\left.y^{2}, x y^{2}-y^{3}, y^{4}\right)$, and so the initial ideal of $I$ is in $I=\left(x^{2}, x y^{2}, y^{4}\right)$. Notice that in $I$ is lexsegment and so always has the strong Lefschetz property by Theorem 4.11. Using [19, Proposition 2.9], the latter implies that $R / I$ also has the strong Lefschetz property, if char $K \neq 2$.

Moreover, we classify the width functions that force monomial ideals to always have the strong Lefschetz property, regardless of characteristic, using Lemma 2.7.

Proposition 4.16. Suppose $w: \mathbf{N}_{0} \rightarrow \mathbf{N}_{0}$ is the width function of some monomial artinian quotient of $R=K[x, y]$. Every monomial artinian quotient $R / I$ such that $w_{R / I}=w$ has the strong Lefschetz property, regardless of the characteristic of $K$, if and only if for every nonnegative integer $d$ we have $0 \leq w(d+1)-w(d) \leq 2$.

Proof. Combine Lemma 2.7 and Theorem 4.11. 
Hence, we can force homogeneous ideals with these width functions to have the strong Lefschetz property.

Corollary 4.17. Suppose $w: \mathbf{N}_{0} \rightarrow \mathbf{N}_{0}$ is the width function of some homogeneous artinian quotient of $R=K[x, y]$. If, for every nonnegative integer $d$, we have $0 \leq w(d+1)-w(d) \leq 2$, then every homogeneous artinian quotient $R / I$ with $w_{R / \operatorname{gin} I}=w$ has the strong Lefschetz property.

Proof. Let $R / I$ be some homogeneous artinian quotient with $w_{R / \operatorname{gin} I}$ $=w$. By Proposition 4.13, $R /$ gin $I$ has the strong Lefschetz property, and so $R / I$ has the strong Lefschetz property by Proposition 4.2.

Acknowledgments. The author would like to thank Juan Migliore and Uwe Nagel for many discussions related to the results contained herein and for help in the presentation of this manuscript.

Further, the author would like to thank Tony Iarrobino for pointing out the earlier results that give Theorem 1.2 and also the anonymous referee for helpful comments that improved the exposition.

\section{REFERENCES}

1. R. Basili and A. Iarrobino, Pairs of commuting nilpotent matrices, and Hilbert function, J. Alg. 320 (2008), 1235-1254.

2. M. Boij, J. Migliore, R. Miró-Roig, U. Nagel and F. Zanello, On the shape of a pure O-sequence, Mem. Amer. Math. Soc. 218 (2012), vii+78 pp.

3. J. Briançon, Description de $\operatorname{Hilb}^{n} \mathbb{C}\{x, y\}$, Invent. Math. 41 (1977), 45-89.

4. K. Chandler, The geometric interpretation of Fröberg-Iarrobino conjectures on infinitesimal neighbourhoods of points in projective space, J. Alg. 286 (2005), 421-455.

5. M. Ciucu, T. Eisenkölbl, C. Krattenthaler and D. Zare, Enumerations of lozenge tilings of hexagons with a central triangular hole, J. Combin. Theor. 95 (2001), 251-334.

6. D. Cook II, The Lefschetz properties of monomial complete intersections in positive characteristic, J. Alg. 369 (2012), 42-58; doi:10.1016/j.jalgebra. 2012.07 .015 .

7. D. Cook II and U. Nagel, Enumerations of lozenge tilings, lattice paths, and perfect matchings and the weak Lefschetz property, preprint (2013); available at arXiv:1305.1314. 
8. I. Gessel and X.G. Viennot, Determinants, paths and plane partitions, preprint (1989); available at http://people.brandeis.edu/ gessel/ homepage/papers/.

9. T. Harima, J. Migliore, U. Nagel and J. Watanabe, The weak and strong Lefschetz properties for Artinian K-algebras, J. Alg. 262 (2003), 99-126.

10. J. Herzog and T. Hibi, Monomial ideals, Grad. Texts Math. 260, SpringerVerlag London, Ltd., London, 2011.

11. A. Iarrobino, Associated graded algebra of a Gorenstein Artin algebra, Mem. Amer. Math. Soc. 107 (1994), viii+115 pp.

12. J. Li and F. Zanello, Monomial complete intersections, the weak Lefschetz property and plane partitions, Discr. Math. 310 (2010), 3558-3570.

13. B. Lindström, On the vector representations of induced matroids, Bull. Lond. Math. Soc. 5 (1973), 85-90.

14. J. Migliore and R. Miró-Roig, Ideals of general forms and the ubiquity of the weak Lefschetz property, J. Pure Appl. Alg. 182 (2003), 79-107.

15. J. Migliore, R. Miró-Roig and U. Nagel, Monomial ideals, almost complete intersections and the weak Lefschetz property, Trans. Amer. Math. Soc. 363 (2011), $229-257$.

16. - On the weak Lefschetz property for powers of linear forms, Alg. Num. Theory 6 (2012), 487-526.

17. J. Migliore and U. Nagel, Survey article: A tour of the weak and strong Lefschetz properties, J. Comm. Alg. 5 (2013), 329-358.

18. J. Migliore and F. Zanello, The Hilbert functions which force the weak Lefschetz property, J. Pure Appl. Alg. 210 (2007), 465-471.

19. A. Wiebe, The Lefschetz property for componentwise linear ideals and Gotzmann ideals, Comm. Alg. 32 (2004), 4601-4611.

20. F. Zanello and J. Zylinski, Forcing the strong Lefschetz and the maximal rank properties, J. Pure Appl. Alg. 213 (2009), 1026-1030.

Department of Mathematics, University of Notre Dame, Notre Dame, IN 46556. Present address: Department of Mathematics and Computer Science, Eastern Illinois University, Charleston, IL 61920

Email address: dwcook@eiu.edu 